Palavras chave: Produção Florestal Desenvolvimento Sustentável Amazônia

Histórico: Recebido 06/03/2012 Aceito 02/02/2015

Keywords: Forest Production Sustainable Development Amazon

Correspondência: viviane_vcorrea@hotmail.com
Viviane Vasconcelos Corrêa', João Ricardo Vasconcellos Gama', Renato Bezerra da Silva Ribeiro', Adriana Ferreira Alves', Diego dos Santos Vieira², Lucas Cunha Ximenes ${ }^{2}$

\section{ESTRUTURA E USO POTENCIAL DE ESPÉCIES ARBÓREAS EM FLORESTA MANEJADA, PA MOJU, SANTARÉM-PARÁ}

RESUMO: Neste estudo, objetivou-se analisar a fitossociologia e o uso múltiplo das espécies arbóreas em floresta manejada na comunidade Actaaia, Assentamento Moju I e II, Santarém-Pará. Foram amostrados 10 ha de floresta manejada, alocando-se, sistematicamente, 20 parcelas de $20 \mathrm{~m} \times 250 \mathrm{~m}$ e mensuradas todas as árvores com DAP $\geq 10 \mathrm{~cm}$. Foram encontradas 128 espécies, pertencentes a 37 famílias botânicas. A diversidade pelo Índice de Shannon foi 4,04, que resultou em $83 \%$ da diversidade máxima da amostra. Cada espécie foi representada, em média, por oito indivíduos e a densidade e área basal foram 341,83 árv $\cdot h^{-1}$ e $18,05 \mathrm{~m}^{2} \cdot \mathrm{ha}^{-1}$, respectivamente. A floresta remanescente apresentou espécies com potencial madeireiro e não madeireiro, para a utilização pela comunidade; a ocorrência de $58 \%$ de espécies com, pelo menos, dois usos revelou a importância de estudar as suas funcionalidades para a melhoria da qualidade de vida dos comunitários, o que deve ser considerado em um plano de manejo florestal comunitário.

\section{STRUCTURE AND POTENTIAL USE OF TREE SPECIES IN A MANAGED FOREST IN PA MOJU, SANTÁREM-PARÁ}

ABSTRACT: The study aimed to analyze the phytosociology and multipurpose tree species use in a managed forest in the Actaaia community, Settlement Moju I and II, Santarém, Pará. 10 ha of managed forest were sampled, systematically allocating 20 plots of $20 \mathrm{~m} \times 250 \mathrm{~m}$, with all trees with $\mathrm{DBH} \geq 10 \mathrm{~cm}$ measured. 128 species belonging to 37 families were found. The Shannon diversity index was 4.04, which resulted in $83 \%$ of the maximum diversity of the sample. Each species was represented on average by eight individuals and the density and basal area were $34 \mathrm{I} .83$ trees $\cdot \mathrm{ha}^{-1}$ and $18.05 \mathrm{~m}^{2} \cdot \mathrm{ha}^{-1}$, respectively. After exploration, the forest contained species with timber and non-timber potential use by the community, with the occurrence of $58 \%$ of species with at least two uses, revealing the importance of studying their features to improve the quality of life of the community, which should be considered in a community forest management plan.
DOI: I0.1590/01047760201521021488
' Universidade Federal do Oeste do Pará - Santarém, Pará, Brasil

2 Universidade Federal dos Vales do Jequitinhonha e Mucuri - Diamantina, Minas Gerais, Brasil 


\section{INTRODUÇÃO}

Nos assentamentos de Reforma Agrária, a infraestrutura e os recursos naturais disponíveis devem ser utilizados de maneira otimizada, por meio de sistemas de produção sustentáveis, baseados na função social da terra e na promoção econômica, social e cultural do trabalhador rural e de seus familiares (BRASIL, 2006).

Nos lotes individuais, dos projetos de assentamento na Amazônia, $80 \%$ da propriedade constitui a reserva legal, que pode receber ações de manejo, tanto para colheita de madeira, como para extração de produtos não madeireiros. Estes últimos, por sua vez, podem ser manejados continuamente, mesmo após colheita madeireira, desde que a área forneça boa produtividade, e que a extração não prejudique a estrutura da população de determinada espécie.

Em 2010, a participação de produtos madeireiros oriundos da extração vegetal brasileira totalizou $R \$ 3,4$ bilhões e os produtos não madeireiros somaram R\$ 778,2 milhões. Valor ainda discreto, baseado, principalmente, na extração de açaí, babaçu, piaçava, erva-mate nativa, pó de carnaúba e castanhado-brasil, que juntos somaram $89 \%$ do total proveniente da atividade extrativista não madeireira (INSTITUTO BRASILEIRO DE GEOGRAFIA E ESTATÍSTICA - IBGE, $2010)$. Esse fato se constrasta com a riqueza de produtos e sua múltipla utilidade que podem ser ofertadas para a sociedade, e que também podem beneficiar às comunidades que detêm desses produtos.

Para demonstrar que a floresta, após colheita florestal madeireira, pode continuar gerando benefícios aos comunitários, por meio do manejo dos PFNMs, o estudo objetivou avaliar o potencial de produtos florestais não madeireiros de espécies arbóreas remanescentes em uma área que sofreu intervenção por meio de manejo florestal.

\section{MATERIAL E MÉTODOS}

\section{Área de estudo}

A comunidade Actaaia (Associação Comunitária dos Trabalhadores da Agricultura e Agropecuária do Igarapé do Anta), integrante do Projeto de Assentamento Moju, está localizada no município de Santarém, à esquerda da rodovia BR 163 , vicinal km $10 \mathrm{I}$, compreendida entre os paralelos $03^{\circ} \mathrm{I} 0^{\prime}$ a $03^{\circ} 20^{\prime}$,

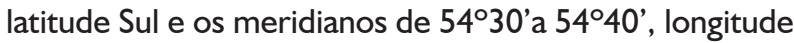

Oeste de Greenwich. Fundada em 1997, abrange uma área de, aproximadamente, 5.760 ha onde vivem 236 pessoas em 52 famílias (GAMA et al., 20I I).

A vegetação é classificada como Floresta Ombrófila Densa de Terra Firme (VELOSO et al., I991). O solo é do tipo latossolo amarelo distrófico (GAMA et al., 20 I I). O clima da região, segundo a classificação de Köppen, é do tipo Am (quente e úmido). A temperatura média anual do município é de $25,6^{\circ} \mathrm{C}$, a umidade relativa apresenta valores acima de $80 \%$ em quase todos os meses do ano e a precipitação pluvial média anual é de $2.000 \mathrm{~mm}$ (FUNDO DE DESENVOLVIMENTO E AÇÃO COMUNITÁRIA - FUNDAC, 2005).

A Área de Manejo Florestal (AMF) abrange uma superfície de $3.845,49$ ha. A madeira foi colhida no ano de 2005, sendo: Manilkara huberi [Ducke] Chevalier (maçaranduba), Mezilaurus itauba Taubert ex Mez. (itaúba) e Tabebuia serratifolia [Vahl] G. Nicholson (ipê amarelo) as espécies de maior volume de extração (GAMA et al., 20I I).

\section{Amostragem e coleta de dados}

O inventário florestal foi realizado em 10 ha de floresta manejada no ano de 2009, ou seja, quatro anos após a colheita de madeira, empregandose amostragem sistemática com inícios aleatórios. As unidades de amostra foram de área fixa de $20 \mathrm{~m} \times 250 \mathrm{~m}$, onde foram mensurados todos os indivíduos que apresentaram diâmetro medido a I,30 $\mathrm{m}$ do solo $(\mathrm{DAP}) \geq 10 \mathrm{~cm}$, considerando três classes de tamanho e suas respectivas subamostragens, a saber: subamostra de $20 \mathrm{~m} \times 50 \mathrm{~m}$, foram mensurados todos os indivíduos com $10 \leq \mathrm{DAP}<30 \mathrm{~cm}$; subamostra de $20 \mathrm{~m} \times 150 \mathrm{~m}$, foram mensurados todos os indivíduos com $30 \mathrm{~cm} \leq$ DAP $<50 \mathrm{~cm}$; e nas amostras de $20 \mathrm{~m}$ x $250 \mathrm{~m}$ foram mensurados os indivíduos com DAP $\geq$ $50 \mathrm{~cm}$.

Nas três classes de tamanho foram anotados os nomes regionais da espécie, diâmetro medido a I,30 $\mathrm{m}$ do solo (DAP) e altura total $(\mathrm{Ht})$. As espécies foram identificadas em campo pelo nome regional e aquelas que suscitaram dúvidas tiveram seu material botânico coletado e a determinação taxonômica feita por especialistas por meio de comparações no herbário da Universidade Federal do Oeste do Pará. A grafia do nome das espécies foi confirmada no banco de dados do Missouri Botanical Garden (20I I).

Foram realizadas entrevistas semiestruturadas com as famílias assentadas e, também, pesquisa 
bibliográfica para se conhecer o uso das espécies encontradas na floresta manejada. Dessa forma, classificaram-se as espécies nos seguintes grupos: madeireiro, medicinal/fármaco, alimentação para a fauna, alimentação humana, construções rústicas, produção de carvão e exsudato/óleo-resinífero, que serviram para sua caracterização como espécies florestais de uso múltiplo.

\section{Análise de dados}

A composição florística foi analisada com base na distribuição dos indivíduos em espécies e famílias. Por meio da lista de espécies, estimou-se a diversidade, utilizando o índice de Shannon-Weaver ( $\left.\mathrm{H}^{\prime}\right)$; a intensidade de mistura das espécies foi obtida a partir do cálculo do quociente de mistura de Jentsch (QM); e a equabilidade de Pielou, todos conforme Brower e Zar (1984).

padrão de distribuição espacial foi calculado pela fórmula de Payandeh (BROWER; ZAR, 1984). Os parâmetros fitossociológicos da estrutura horizontal (densidade, frequência e dominância) e vertical (valor de importância ampliado) foram calculados, segundo Mueller-Dombois e Ellenberg (1974).

Para a avaliação do potencial das espécies fornecedoras de PFNM foram avaliados os aspectos de estrutura fitossociológica, além das quantidades e tipos de uso identificados nas entrevistas com os comunitários.

A tabulação e o processamento de dados foram realizados por meio do software Microsoft Excel 2010.

\section{RESULTADOS E DISCUSSÃO}

\section{Composição florística}

Foram mensuradas 97I árvores, pertencentes a 128 espécies botânicas. A amostra apresentou índice de diversidade de Shannon-Weaver igual a 4,04, considerado alto, sendo similar ao encontrado por Salomão et al. (2007), avaliando florestas exploradas em Altamira e Vitória do Xingu ( $\mathrm{H}^{\prime}=4,04$ e H'= 3,86, respectivamente). Almeida et al. (20I2) em uma área manejada na comunidade Santo Antônio do PA Moju, oeste do Pará, encontrou H'=4,39.

$\mathrm{O}$ índice de Equabilidade de Pielou (J) indicou que $83 \%$ da diversidade máxima possível foi alcançada na amostra. $\mathrm{O}$ quociente de mistura de Jentsch (QM) foi de I:8, ou seja, cada espécie foi representada, em média, por oito indivíduos (Tabela I). Almeida et al. (2012) encontrou $\mathrm{QM}=1: 7$, em floresta manejada em Santarém. O resultado representou boa heterogeneidade florística, concordando com Finol (1975), ao explicar que em florestas tropicais o QM seria de, aproximadamente, nove indivíduos por espécie.

TABELA 1 Índices estruturais e de diversidade para indivíduos com DAP $\geq 10 \mathrm{~cm}$ amostrados em floresta manejada, Comunidade Actaaia, BR 163, Santarém-PA.

TABLE 1 Structural and diversity indexes for individuals with $\mathrm{DBH} \geq 10 \mathrm{~cm}$, sampled in a managed forest, Actaaia Community, BR 163, Santarém-PA.

\begin{tabular}{lc}
\hline Parâmetro & Floresta Manejada \\
\hline Amostra (ha) & 10 \\
Número de Famílias & 34 \\
Riqueza de Espécies (S) & 128 \\
Densidade Absoluta (ind.ha-1) & 341,8 \\
Área Basal (m².ha-1) & 18,05 \\
Diversidade Máxima (Hmáx) & 4,85 \\
Índice de Shannon-Weaver (H') & 4,04 \\
Equabilidade de Pielou (J) & 0,83 \\
Coeficiente de Mistura de Jentsch (QM) & 7,6 \\
\hline
\end{tabular}

Para Machado (2008), o manejo de PFNMs é favorecido quando os individuos ocorrem de forma agrupada, uma vez que a logística para coleta é facilitada. Quanto ao Padrão de Distribuição Espacial (PDE) 28\% das espécies identificadas apresentaram distribuição aleatória, como espécies importantes quanto ao seu potencial de uso, tem-se: Cecropia pachystachya Trec., Bertholletia excelsa H. B. K., Hymenaea courbaril L., Caryocar villosum (Aubl.) Pers. Outros 19\% foram consideradas com tendência ao agrupamento, destacase nesse grupo Carapa guianensis Aubl.

As espécies que ocorreram de maneira agrupada totalizaram $27 \%$, dentre estas se destacaram Licania kunthiana Hook.f., Tabebuia serratifolia (Vahl) G. Nicholson, Apuleia leiocarpa (Vogel) J.F.Macbr., Trattinnickia glaziovii Swart, Diplotropis purpúrea (Rich.) Amshoff, Glicoxylon pedicellatum Ducke e Neea floribunda Poepp. \& Endl. que possuem uso medicinal. Já, as espécies que ocorreram em apenas uma parcela, consideradas com baixa densidade local, totalizaram $26 \%$, entre estas se destacam Brosimum pubescens Taub., Hevea brasiliensis (Willd. ex Juss.) M. Arg., Copaifera reticulata Ducke e Aniba canellila Mez., que apresentaram o maior número de usos entre as espécies encontradas. 


\section{Estrutura Horizontal}

Adensidade foide $34 \mathrm{I}, 83$ ind $\cdot \mathrm{ha}^{-1}$, representando uma área basal de $18,05 \mathrm{~m}^{2} \cdot \mathrm{ha}^{-1}$. Dentre as 128 espécies identificadas, 67 apresentaram densidade absoluta (DA) maior ou igual a uma árvore por hectare (Tabela 2).
As 10 espécies mais abundantes (DA > 6,5 árv'ha ${ }^{-1}$ ) foram Pouteria cladantha, Eschweilera paniculata, Licania kunthiana, Richardella macrophylla, Pouteria guianensis, Inga sp., Inga auristellae, Neea floribunda, Poecilanthe sp., Alexa grandiflora que, juntas, representam $44,43 \%$ da densidade total.

TABELA 2 Estimativa dos parâmetros fitossociológicos para as espécies com VIA $\geq 1,4$ inventariadas em floresta não manejada, Comunidade Actaaia, BR 163, Santarém-PA. Em que: FA = frequência absoluta; DA = densidade absoluta; DoR = dominância relativa; VIA = valor de importância ampliado em porcentagem.

TABLE 2 Horizontal structure parameter estimates for the species with VIA $\geq 1.4$, inventoried in unmanaged forests, Actaaia Community, BR 163, Santarém-PA. Where: FA = absolute frequency; DA = absolute density; DoR= relative dominance; VIA = Expanded value of importance in percentage.

\begin{tabular}{|c|c|c|c|c|c|c|}
\hline $\mathrm{N}^{\circ}$ & Espécie & $\mathrm{P}$ & FA & DA & DoR & VIA \\
\hline 1 & Pouteria cladantha Sandwith & $A G$ & 95 & 39,8 & 9,09 & 9,07 \\
\hline 2 & Eschweilera paniculata Miers. & TA & 95 & 21,4 & 5,47 & 5,46 \\
\hline 3 & Licania incana Aubl. & AG & 80 & 15,8 & 2,63 & 3,84 \\
\hline 4 & Pouteria macrophylla (Lam.) Eyma & AG & 45 & 14,0 & 2,80 & 3,28 \\
\hline 5 & Inga sp. & TA & 60 & 12,0 & 1,81 & 2,88 \\
\hline 6 & Alexa grandiflora Ducke & AG & 65 & 7,3 & 4,87 & 2,87 \\
\hline 7 & Inga alba (Sandw.) Willd. & AG & 60 & 11,6 & 1,98 & 2,64 \\
\hline 8 & Franchetella anibaefolia (A.C. Smith.) Aubr. & AG & 30 & 12,1 & 1,66 & 2,45 \\
\hline 9 & Neea floribunda Poepp. \& Endl. & AG & 60 & 9,8 & 1,66 & 2,24 \\
\hline 10 & Cordia nodosa Lam. & A & 70 & 5,4 & 2,48 & 1,96 \\
\hline 11 & Manilkara huberi (Ducke) Chevalier & $A G$ & 55 & 3,07 & 4,09 & 1,88 \\
\hline 12 & Pouteria bilocularis (H. Winkler) Baehni & TA & 55 & 4,9 & 2,46 & 1,84 \\
\hline 13 & Duguetia cadaverica Huber & TA & 45 & 6,5 & 0,89 & 1,68 \\
\hline 14 & Eperua schomburgkiana Benth. & TA & 45 & 6,8 & 0,86 & 1,67 \\
\hline 15 & Geissospermum vellosii Allem. & A & 60 & 3,5 & 2,18 & 1,65 \\
\hline 16 & Glicoxylon pedicellatum Ducke & $A G$ & 40 & 5,4 & 1,63 & 1,61 \\
\hline 17 & Licaria guianensis Aublet & TA & 45 & 6,0 & 0,92 & 1,60 \\
\hline 18 & Eperua bijuga Mart. & TA & 45 & 7,5 & 0,53 & 1,59 \\
\hline 19 & Ocotea sp. & $A G$ & 30 & 6,1 & 1,36 & 1,51 \\
\hline 20 & Guatteria poeppigiana Mart. & TA & 40 & 5,7 & 0,76 & 1,43 \\
\hline 21 & Sclerolobium sp. & TA & 45 & 3,8 & 1,06 & 1,26 \\
\hline 22 & Ocotea neesiana (Miq.) Kosterm. & TA & 35 & 4,7 & 0,73 & 1,21 \\
\hline 23 & Rudgea cornibolia (H.B.K.) Standl. & $A G$ & 30 & 5,1 & 0,68 & 1,19 \\
\hline 24 & Protium polybotryum Engl. & $A G$ & 30 & 4,7 & 0,58 & 1,17 \\
\hline 25 & Sloanea nitida G. Don & TA & 35 & 4,7 & 0,52 & 1,12 \\
\hline 26 & Jacaratia spinosa (Aubl.) A. DC. & $A G$ & 15 & 5,3 & 0,80 & 1,12 \\
\hline 27 & Elizabetha paraensis Ducke & $A G$ & 30 & 4,5 & 0,75 & 1,11 \\
\hline 28 & Sclerolobium melanocarpum Ducke & TA & 35 & 2,6 & 1,66 & 1,10 \\
\hline 29 & Swartzia aptera D.C. & A & 40 & 3,5 & 0,90 & 1,06 \\
\hline 30 & Rinorea flavescens (Kuntz.) Aubl. & TA & 35 & 4,5 & 0,37 & 1,06 \\
\hline \multicolumn{3}{|c|}{ Subtotal } & 1450 & 248,2 & 58,18 & 64,53 \\
\hline \multicolumn{3}{|c|}{ Outras } & 1300 & 93,6 & 41,82 & 35,47 \\
\hline \multicolumn{3}{|c|}{ Total } & 2750 & 341,8 & 100 & 100 \\
\hline
\end{tabular}


Considerando espécies com maiores valores de densidade absoluta (DA) da floresta e que também se mostraram bem distribuídas na área (60 a 100\% de frequência), destacaram-se Pouteria cladantha, Eschweilera paniculata, Licania kunthiana, Alexa grandiflora, Inga sp., Inga auristellae, Neea floribunda (Tabela 2).

As 10 espécies com maior área basal (DoA $\left.\geq 0,38 \mathrm{~m}^{2} \cdot \mathrm{ha}^{-1}\right)$, foram Pouteria cladantha, Eschweilera paniculata, Alexa grandiflora, Manilkara huberi, Richardella macrophylla, Licania kunthiana, Cordia nodosa, Pouteria bilocularis, Geissospermum sericeum, Parkia multijuga que, juntas, representaram $38,18 \%$ de dominância total (Tabela 2).

As 30 espécies mais importantes, de acordo com - VIA, representaram $64,53 \%$ do VIA total da amostra, sendo que a Pouteria cladantha foi a mais importante, seguida por Eschweilera paniculata, Licania kunthiana, Richardella macrophylla e Inga spl. (Tabela 2).

Quanto ao uso das espécies, aquelas com os maiores valores de VIA (VIA $\geq 1,96)$ foram: Pouteria cladanta - espécie destinada à produção de energia e seus frutos são apreciados pela fauna; Eschweilera paniculata - a madeira dessa espécie é utilizada em serraria, seus frutos servem para a alimentação da fauna e sua entrecasca é utilizada na fabricação de cordas. Licania kunthiana - os frutos são consumidos pela fauna local e têm propriedades medicinais; Richardella macrophylla - utilizada em serraria e seus frutos são utilizados como alimento humano e da fauna; Inga sp. - seus frutos são fonte de alimento para a fauna e para o homem. As plantas desse gênero são pioneiras, utilizadas em plantios mistos em áreas ciliares degradadas, pode ser usada, ainda, no paisagismo, uma vez que também cresce em terrenos bem drenados; a madeira pode ser usada para o fabrico de caixotaria, lápis e brinquedos (LORENZI, 2002).

Alexa grandiflora - a sua madeira é desdobrada em serraria e seus frutos alimentam a fauna; Inga auristellae - sua madeira é utilizada em serraria, seus frutos servem para o alimento humano e animal e é indicado para a produção de energia; Pouteria guianensis sua madeira é utilizada em serraria, seus frutos são fonte de alimento para os animais, e é destinada à produção de energia; Neea floribunda - a madeira dessa espécie é utilizada em serraria e seus frutos são utilizados como alimento humano e animal; Cordia nodosa- seus frutos são utilizados na alimentação da fauna. Dentre as demais espécies que ocuparam as primeiras posições do VIA, destacam-se Manilkara huberi, uma das espécies de maior interesse econômico atualmente, pois são inúmeros os usos de sua madeira, sendo o principal em construção civil (HIRAl et al., 2008; PINHEIRO et al., 2007).

\section{Grupos de Uso}

O diagnóstico do potencial florestal de um ecossistema baseia-se, principalmente, nas informações sobre o valor desses produtos para a sociedade (GAMA et al., 2007). Na área manejada $99,8 \%$ das espécies apresentaram pelo menos um único uso. As espécies com maior alternativa de uso foram Brosimum rubescens, Hymenaea courbaril, Caryocar villosum, com cinco usos; Carapa guianensis, Bertholletia excelsa, Copaifera reticulata com quatro usos cada.

A espécie Brosimum rubescens (Amapá) pertencente à família Moraceae, é uma espécie monóica, forma florestas monodominantes, com indivíduos de 20 a $35 \mathrm{~m}$ de altura e 20 a $50 \mathrm{~cm}$ de diâmetro, com fuste reto e cilíndrico na fase madura (MARIMON; FELFILI, 2006). A espécie é semidecídua, perdendo suas folhas durante todo o ano, com pico na época da seca (MARIMON et al., 2008).

O exsudato do Amapá é utilizado na medicina popular para tratamento de tuberculose, gripe, tosse, garganta, diabete e estômago (SCUDELLER et al., 2009). A madeira é utilizada na carpintaria naval e civil, além do fabrico de móveis de esmerado acabamento, por conta de sua beleza, apesar de seu elevado peso (GONZAGA, 2006).

A espécie Hymenaea courbaril (Jatobá) pertence à família Fabaceae, é uma espécie semidecídua, heliófila, pouco exigente às condições de fertilidade e umidade do solo. A madeira é dura ao corte, possui média resistência ao ataque de agentes xilófagos sob condições naturais, sendo recomendada na construção naval e na construção civil (GONZAGA, 2006).

Da casca dessa árvore faz-se chá bastante utilizado na medicina popular, a seiva também é usada como combustível, remédio, verniz natural, polimento e impermeabilizador de canoa. Quando exposta ao ar, essa seiva forma uma resina, conhecida como jutaicica, normalmente, encontrada na base do tronco, esta é utilizada para alívio de dor de estômago, e é queimada para inalação em casos de resfriados, além de ser um ótimo verniz natural. As folhas possuem uma substância química, terpenóide, que mata fungos, repele as saúvas e lagartas, apresenta efeitos tóxicos. As sementes são empregadas na fabricação de jóias e outros objetos artesanais (SHANLEY; MEDINA, 2005).

A espécie Caryocar villosum (piquiá) é uma espécie de uso variado, podendo ser usada madeira, fruto e óleo extraído das sementes. A madeira é considerada de alta densidade, possuindo grande resistência, por isso é recomendada para carpintaria 
naval, construção civil (GONZAGA, 2006; SHANLEY; MEDINA, 2005).

Seus frutos são bastante apreciados pelas comunidades na Amazônia, depois de cozido, a polpa é utilizada como alimento. Cada fruto possui entre uma e quatro sementes, a casca é esverdeada, a polpa é amarelada (PESCE, 2009; SOUSA et al., 2005). As flores são apreciadas pelos animais; a casca dos frutos apresenta elevado teor de tanino, que pode substituir a noz de galha na preparação da tinta para escrever, para tingir tecidos (SHANLEY; MEDINA, 2005), além de tingir redes de pesca. $O$ óleo de pequiá é utilizado na culinária local, mas, ultimamente, tem sido alvo de pesquisas sobre biocombustíveis.

A espécie Carapa guianensis (andiroba) é uma espécie que apresenta uso múltiplo, a cor de sua madeira é parecida com o mogno (Swietenia macrophylla King.) (COSTA et al., 2007; GONZAGA, 2006). O seu fruto é uma cápsula globosa e deiscente, com cinco a nove sementes. Por meio destas obtém-se um óleo utilizado na medicina tradicional com funções cicatrizantes, antiinflamatórias, antihelmínticas, e no mercado internacional na preparação de sabão, cosméticos e velas, e na indústria de cosméticos, além da casca que tem uso medicinal contra febre, vermes, bactérias e tumores (SHANLEY; MEDINA, 2005).

A espécie Bertholletia excelsa (castanha-dopará) é uma espécie que ocorre nas áreas altas de terra firme. Os frutos, conhecidos como ouriço, são cápsulas lenhosas que possuem em seu interior em média 15 sementes (HADDAD; BONELLI, 2006). Essas sementes são amêndoas oleaginosas que apresentam alto valor nutritivo, rica em proteínas, e em outros nutrientes como o selênio, que vem sendo referido na prevenção de câncer e de doenças cardiovasculares (GLÓRIA; REGITANO-ARCE, 2000; SOUZA; MENEZES, 2004). $O$ ouriço pode ser utilizado na confecção de artesanato ou como combustível para a defumação da borracha, enquanto as cascas das sementes são utilizadas para a fabricação de ração animal (HADDAD; BONELLI, 2006).

$O$ produto é bastante abundante, tendo em vista a alta densidade e a produtividade da castanheira. Apresenta grande importância econômica, possuindo sólida demanda de mercado e sua coleta é de baixo impacto ambiental, sendo considerada, assim, uma espécie essencial para o desenvolvimento de comunidades tradicionais na região amazônica (HADDAD; BONELLI, 2006; TONINI et al., 2008).

A espécie Copaifera reticulada (copaíba) pertence ao gênero copaifera L., constituído por árvores com altura de 15 a 40 metros, desta é possível explorar a madeira, utilizada na carpintaria e construção civil, e fabricação de tábuas (GONZAGA, 2006; SHANLEY; MEDINA, 2005) e seus frutos são apreciados pelos animais silvestres, além de fornecer um óleo essencial bastante utilizado na medicina popular. $O$ óleo também vem sendo utilizado como anticoncepcional; como combustível, substituindo a função do tradicional diesel nas lamparinas (GUERRA, 2008).

A floresta remanescente apresentou bom potencial de aproveitamento, pois do total de espécie, $63,3 \%$ possuem aproveitamento madeireiro. Além disso, $58 \%$ das espécies encontradas apresentam, pelo menos, dois usos, fato que revela a importância de relacionar as funcionalidades das espécies de uso múltiplo à qualidade de vida dos comunitários, o que deve ser considerado no planejamento do manejo florestal.

Foram encontradas $21,1 \%$ de espécies que são utilizadas na medicina popular; $58,6 \%$ de espécies que seus frutos servem de alimento para a fauna silvestre; $15,6 \%$, alimento humano; $14,8 \%$ são espécies que exsudam algum óleo-resina, ou seiva, foram incluídas, ainda nessa categoria, árvores como Carapa guianensis, cujas sementes produzem óleo; 7,8\% das espécies são utilizadas para construções rústicas; e 7,8\% podem ser aproveitadas para carvão ou produção de lenha.

Durante o planejamento florestal, é necessário que não se observe apenas $\circ$ valor madeireiro da floresta, mas, também, deve-se priorizar $\circ$ acesso contínuo a determinadas espécies de uso múltiplo pelos comunitários (ALMEIDA, 20I0). Dentre as espécies que devem ser mantidas na área para serem utilizadas exclusivamente para $\bigcirc$ fornecimento de PFNM, destacam-se Carapa guianensis, Caryocar villosim, Copaifera reticulada, Aniba canellila, Bertholletia excelsa, Brosimum rubescens e Hymenaea courbaril. Algumas dessas espécies já apresentam uma pequena escala de comercialização na comunidade, como é o caso da Carapa guianensis e Brosimum rubescens.

\section{CONCLUSÕES}

Aocorrência de mais da metade de espécies com, pelo menos, dois usos revelou a importância de estudar as suas funcionalidades para a melhoria da qualidade de vida dos comunitários, o que deve ser considerado em um plano de manejo florestal comunitário.

Pode-se recomendar que espécies como Carapa guianensis, Caryocar villosum, Aniba canellila, Copaifera reticulata e Hymenaea courbaril sejam removidas da 
lista de espécies de corte para fins madeireiros, devido fornecerem produtos de interesse ao mercado de produtos florestais não madeireiros.

$\mathrm{Na}$ seleção de espécies para colheita deve ser incluído o critério de uso múltiplo das mesmas, escolhendo-se, assim, a melhor destinação socioeconômica para as espécies.

\section{REFERÊNCIAS}

ALMEIDA, L. S. Produtos florestais não madeireiros em área manejada: análise de uma comunidade na região de influência da BR I63, Santarém, Estado do Pará. 2010. 128 f. Dissertação (Mestrado em Ciências Florestais) Universidade Federal Rural da Amazônia, Belém, 2010.

ALMEIDA, L. S.; GAMA, J. R. V.; OLIVEIRA, F. A.; CARVALHO, J. O. P.; GONÇALVES, D. C. M.; ARAÚJO, G. C. Fitossociologia e uso múltiplo de espécies arbóreas em floresta manejada, comunidade Santo Antônio, Município de Santarém, Estado do Pará. Acta Amazônica, Manaus, v. 42, n. 2, p. 183-191, 2012.

BRASIL. Conselho Nacional do Meio Ambiente. Resolução $n^{\circ}$ 387, de 27 de dezembro de 2006. Estabelece procedimentos para o Licenciamento Ambiental de Projetos de Assentamentos de Reforma Agrária, e dá outras providências. Brasília, 2006. 15 p.

BROWER, J. E.; ZAR, J. H. Field and laboratory methods for general ecology. $2^{\text {nd }}$ ed. Dubuque: W. M. C. Brown, 1984. $226 \mathrm{p}$.

COSTA, G. F; SALLA, L.; MARENCO, R. A. Trocas gasosas e potencial hídrico em Carapa guianensis Aubl. Revista Brasileira de Biociências, Porto Alegre, v. 5, n. 2, p. I56I58, jul. 2007. Suplemento.

FINOL, U. H. La silvicultura en la Orinoquia Venezolana. Revista Forestal Venezolana, Mérida, v. 18, n. 25, p. 37II4, 1975.

FUNDO DE DESENVOLVIMENTO E AÇÃO COMUNITÁRIA. Plano de desenvolvimento sustentável Moju I e II. Santarém, 2005. 153 p.

GAMA, J. R. V.; MORAES, A.; CARVALHO, A. N.; RIBEIRO, A. S. S.; SANTOS, C. A. A.; MONTEIRO, R.; GOCH, Y. G. F. Estudo de impacto ambiental e proposta de desenvolvimento sustentável para o assentamento Moju I e II, município de Santarém-Pa. Santarém: UFOPA, 20II. 235 p. Relatório final, CNPq I5/2007, nº 485077I 2007-8.

GAMA, J. R. V.; SOUZA, A. L.; CALEGÁRIO, N.; LANA, G. C. Fitossociologia de duas fitocenoses de floresta ombrófila aberta no município de Codó, Estado do Maranhão. Revista Árvore, Viçosa, v. 3I, n. 3, p. 465-477, 2007.
GLÓRIA, M. M.; REGITANO-ARCE, M. A. B. d'. Concentração e isolado protéico de torta de castanha-do-pará: obtenção e caracterização química e funcional. Ciências e Tecnologia e Alimentos, Campinas, v. 20, n. 2, p. 240-245, 2000.

GONZAGA, A. L. Madeira: uso e conservação. Brasília: IPHAN/MONUMENTA, 2006. 246 p.

GUERRA, F. G. P. Q. Contribuição dos produtos florestais não madeireiros na geração de renda na floresta nacional do Tapajós, Pará. 2008. 133 p. Dissertação (Mestrado em Ciências Florestais) - Universidade Federal do Paraná, Curitiba, 2008.

HADDAD, C. J.; BONELLI, M. F. Projeto Castanha-doBrasil Amapá. Macapá: Fundação Getúlio Vargas, 2006.

HIRAI, E. H.; CARVAlHO, J. O. P.; PINHEIRO, K. A. O. Estrutura da população de maçaranduba (Manilkara huberi Standley) em 84 ha de floresta natural na Fazenda Rio Capim, Paragominas, PA. Revista de Ciências Agrárias, Belém, n. 49, p. 65-76, 2008.

INSTITUTO BRASILEIRO DE GEOGRAFIA E ESTATÍ́STICA. Produção da extração vegetal e da silvicultura. Rio de Janeiro, 2010. 52 p.

LORENZI, H. Árvores brasileiras: manual de identificação e cultivo de plantas arbóreas nativas do Brasil. 4. ed. Nova Odessa: Instituto Plantarum, 2002. v. I, 384 p.

MACHADO, F. S. Manejo de produtos florestais não madeireiros: um manual com sugestões para $\circ$ manejo participativo em comunidades da Amazônia. Rio Branco: PESACRE; CIFOR, 2008. 105 p.

MARIMON, B. S.; FELFILI, J. M. Chuva de sementes em uma floresta monodominante de Brosimum rubescens Taub. e em uma floresta mista adjacente no Vale do Araguaia-MT. Acta Botanica Brasilica, Porto Alegre, v. 20, n. 2, p. 423432, set. 2006.

MARIMON, B. S.; FELFILI, J. M.; MARIMON-JÚNIOR, B. H.; FRANCO, A. C.; FAGG, C. W. Desenvolvimento inicial e partição de biomassa de Brosimum rubescens Taub. (Moraceae) sob diferentes níveis de sombreamento. Acta Botanica Brasilica, Porto Alegre, v. 22, n. 4, p. 94I953, 2008.

MISSOURI BOTANICAL GARDEN. Nomenclatural. Disponível em: <http://mobot.mobot.org/cgi-bin/search_ vast $>$. Acesso em: 16 mar. $201 \mathrm{l}$.

MUELLER-DOMBOIS, D.; ELLENBERG, G. H. Aims and methods of vegetation ecology. New York: Willey, 1974. $546 \mathrm{p}$.

PESCE, C. Oleaginosas da Amazônia. 2. ed. Belém: Núcleo de Estudos Agrários e Desenvolvimento Rural, 2009. 334 p. 
PINHEIRO, K. A. O.; CARVALHO, J. O. P. de; QUANZ, B.; FRANCEZ, L. M. de B. SCHWARTZ, G. Fitossociologia de uma área de preservação permanente no leste na Amazônia: indicação de espécies para recuperação de áreas alteradas. Revista Floresta, Curitiba, v. 37, n. 2, p. I75-187, 2007.

SALOMÃO, R. P.; VIEIRA, I. C. G.; SUEMITSU, C.; ROSA, N. A.; ALMEIDA, S. S.; AMARAL, D. D.; MENEZES, M. P. M. As florestas de Belo Monte na grande curva do rio Xingu, Amazônia Oriental. Boletim do Museu Paraense Emílio Goeldi. Ciências Naturais, Belém, v. 2, n. 3, p. 57-153, set./dez. 2007.

SCUDELLER, V. V.; VEIGA, J. B.; ARAÚJO-JORGE, L. H. de. Etnoconhecimento de plantas de uso medicinal nas comunidades São João do Tupé e Central: reserva de desenvolvimento sustentável do Tupé. In: SANTOS-SILVA, E. N.; SCUDELLER, V. V. (Org.). Biotupé: meio físico, diversidade biológica e sociocultural do Baixo Rio Negro, Amazônia Central. Manaus: UEA, 2009. v. 2, p. 185-199.

SHANLEY, P.; MEDINA, G. Frutíferas e plantas úteis na vida amazônica. Belém: CIFOR, 2005. 300 p.
SOUZA, M. L.; MENEZES, H. C. Processamento de amêndoas e torta de castanha do Brasil e farinha de mandioca: parâmetros de qualidade. Ciências e Tecnologia e Alimentos, Campinas, v. 20, n. 2, p. 120-128, jan./mar. 2004.

SOUZA, T. M. H. de; SANTIAGO, D. K.; REGIANI, A. M.; NASCIMENTO, M. G. da S.; NASCIMENTO, I. A. do; SARTORI, R. A.; MORAIS, L. C. de. Estudo do potencial do fruto de pequi (Caryocar villosum) para obtenção de óleo e síntese de biodiesel. In: REUNIÃO ANUAL DA SOCIEDADE BRASILEIRA DE QUÍMICA, 25., 2005, Belo Horizonte. Anais... Belo Horizonte: Sociedade Brasileira de Química, 2005. I CD-ROM.

TONINI, H.; COSTA, P. da; KAMINSKI, P. E. Manejo de produtos florestais não madeireiros na Amazônia (Castanheira-do-Brasil) resultados de pesquisa. Boa Vista: EMBRAPA Roraima, 2008. 3 I p. (Boletim de Pesquisa e Desenvolvimento, 2).

VELOSO, H. P.; RANGEL FILHO, A. L. R.; LIMA, J. C. A. Classificação da vegetação brasileira, adaptada a um sistema universal. Rio de Janeiro: IBGE, | 99I. I23 p. 